\title{
Some Curvature Properties of D-conformal Curvature Tensor on LP-Sasakian Manifolds
}

\author{
Riddhi Jung Shah \\ Department of Mathematics, Janata Campus, Dang \\ Nepal Sanskrit University \\ E-mail: shahrjgeo@gmail.com
}

\begin{abstract}
This paper deals with the study of geometry of Lorentzian para-Sasakian manifolds. We investigate some properties of D-conformally flat, D-conformally semi-symmetric, $\xi$-D-conformally flat and $\varphi$-D-conformally flat curvature conditions on Lorentzian para-Sasakian manifolds. Also it is proved that in each curvature condition an LP-Sasakian manifold $\left(M^{n}, g\right)(n>3)$ is an $\eta$-Einstein manifold.
\end{abstract}

Keywords: D-conformal curvature tensor, $\eta$-Einstein manifold, Lorentzian para-Sasakian manifold, $\varphi$-Dconformally flat, semi-symmetric.

\section{INTRODUCTION}

Matsumoto (1989) introduced the notion of Lorentzian para-Sasakian manifold. Then Mihai and Rosca (1992) introduced the same notion independently and they obtained many interesting results on this manifold. Ikawa and Erdogan (1996) and Ikawa and Jun (1997) studied Sasakian manifolds with Lorentzian metric and obtained several results in this manifold. De et al. (2002) introduced the notion of Lorentzian paraSasakian manifold with a coefficient $\alpha$ which generalizes the notion of LP-Sasakian manifold. Lorentzian para-Sasakian manifolds have also been studied by Matsumoto and Mihai (1988), De et al. (1999), Shaikh and Biswas (2004), Tripathi and De (2001), Bagewadi et al. (2008) etc.

A Riemannian or pseudo-Riemannian manifold is said to be semi-symmetric (Szabo, 1982) if $R(X, Y) . R=0$, where $\mathrm{R}(\mathrm{X}, \mathrm{Y})$ denotes the derivation in tensor algebra at each point of the manifold. In this paper, some derivation conditions on LP-Sasakian manifolds are studied. After preliminaries, some results on D-conformally flat LPSasakian manifold, D-conformally semi-symmetric LPSasakian manifold, $\xi$-D-conformally flat LP-Sasakian manifold and $\varphi$-D-conformally flat LP-Sasakian manifold are investigated. In each curvature condition it is proved that a manifold is $\eta$-Einstein.

\section{PRELIMINARIES}

A differentiable manifold of dimension $n$ is called Lorentzian para-Sasakian manifold (briefly LP-Sasakian manifold) (Matsumoto 1989, Mihai \& Rosca 1992), if it admits a $(1,1)$ tensor field $\varphi$, a contravariant vector field $\xi$, a 1-form $\eta$ and a Lorentzian metric $g$ which satisfy

$$
\begin{aligned}
& \eta(\xi)=-1, \\
& \varphi^{2}(X)=X+\eta(X) \xi \\
& g(\varphi X, \varphi Y)=g(X, Y)+\eta(X) \eta(Y), \\
& \nabla_{X} \xi=\varphi X, \\
& \left(\nabla_{X} \varphi\right)(Y)=g(X, Y) \xi+\eta(Y) X+2 \eta(X) \eta(Y) \xi
\end{aligned}
$$

where $\nabla$ denotes the covariant differentiation with respect to the Lorentzian metric $g$.

It can be easily seen that in an LP-Sasakian manifold the following results hold:

$$
\begin{aligned}
& \varphi \xi=0, \eta \circ \varphi=0, \\
& \operatorname{rank} \varphi=n-1 .
\end{aligned}
$$

Again, if we put

$$
\Omega(X, Y)=g(X, \varphi Y)=g(\varphi X, Y),
$$

for any vector fields $X$ and $Y$, then the tensor field $\Omega(X, Y)$ is a symmetric $(0,2)$ tensor field (Matsumoto, 1989). Also since the 1 -form $\eta$ is closed in an LPSasakian manifold, we have (Matsumoto, 1989, De et al. 1999) 


$$
\left\{\begin{array}{l}
\left(\nabla_{X} \eta\right)(Y)=\Omega(X, Y)=g(X, \varphi Y), \\
\Omega(X, \xi)=0,
\end{array}\right.
$$

for any vector fields $X$ and $Y$.

An LP-Sasakian manifold $M$ is said to be $\eta$-Einstein if its Ricci tensor $S$ of type $(0,2)$ is of the form

$$
S(X, Y)=\alpha g(X, Y)+\beta \eta(X) \eta(Y),
$$

for any vector fields $X$ and $Y$, where $\alpha$ and $\beta$ are smooth functions on the manifold. In particular, if $\beta=0$, then the manifold is said to be an Einstein manifold.

Also, in an $n$-dimensional LP-Sasakian manifold, the following relations hold (Matsumoto \& Mihai, 1988, De et al., 1999)

$$
\begin{aligned}
& \eta(R(X, Y) Z)=g(Y, Z) \eta(X)-g(X, Z) \eta(Y), \\
& R(\xi, X) Y=g(X, Y) \xi-\eta(Y) X, \\
& \mathrm{R}(\mathrm{X}, \mathrm{Y}) \xi=\eta(\mathrm{Y}) \mathrm{X}-\eta(\mathrm{X}) \mathrm{Y}, \\
& R(\xi, X) \xi=X+\eta(X) \xi \text {, } \\
& S(X, \xi)=(n-1) \eta(X) \text {, } \\
& S(\varphi X, \varphi Y)=S(X, Y)+(n-1) \eta(X) \eta(Y),
\end{aligned}
$$

for any vector fields $X, Y, Z$, where $R(X, Y) Z$ is the Riemannian curvature tensor. The D-conformal curvature tensor on a Riemannian manifold $\left(M^{n}, g\right)(n>4)$ is defined as (Chuman, 1983)

$\mathrm{B}(\mathrm{X}, \mathrm{Y}) \mathrm{Z}=\mathrm{R}(\mathrm{X}, \mathrm{Y}) \mathrm{Z}+\frac{1}{\mathrm{n}-3}[\mathrm{~S}(\mathrm{X}, \mathrm{Z}) \mathrm{Y}-\mathrm{S}(\mathrm{Y}, \mathrm{Z}) \mathrm{X}+\mathrm{g}(\mathrm{X}, \mathrm{Z}) \mathrm{QY}$

$-\mathrm{g}(\mathrm{Y}, \mathrm{Z}) \mathrm{QX}-\mathrm{S}(\mathrm{X}, \mathrm{Z}) \eta(\mathrm{Y}) \xi+\mathrm{S}(\mathrm{Y}, \mathrm{Z}) \eta(\mathrm{X}) \xi$

$-\eta(\mathrm{X}) \eta(\mathrm{Z}) \mathrm{QY}+\eta(\mathrm{Y}) \eta(\mathrm{Z}) \mathrm{QX}]$

$-\frac{\mathrm{K}-2}{\mathrm{n}-3}[\mathrm{~g}(\mathrm{X}, \mathrm{Z}) \mathrm{Y}-\mathrm{g}(\mathrm{Y}, \mathrm{Z}) \mathrm{X}]+\frac{\mathrm{K}}{\mathrm{n}-3}[\mathrm{~g}(\mathrm{X}, \mathrm{Z}) \eta(\mathrm{Y}) \xi$

$-\mathrm{g}(\mathrm{Y}, \mathrm{Z}) \eta(\mathrm{X}) \xi+\eta(\mathrm{X}) \eta(\mathrm{Z}) \mathrm{Y}-\eta(\mathrm{Y}) \eta(\mathrm{Z}) \mathrm{X}]$

where $K=\frac{r+2(n-1)}{n-2}, R$ is the curvature tensor, $S$ is the Ricci tensor and $r$ is the scalar curvature.

\section{RESULTS AND DISCUSSION \\ Theorem 1}

A D-conformally flat LP-Sasakian manifold $\left(M^{n}, g\right)$ is an $\eta$-Einstein manifold.

Proof: Let us consider an $n$-dimensional LP-Sasakian manifold $\left(M^{n}, g\right)$. For D-conformally flat LP-Sasakian manifold, we have
$B(X, Y) Z=0$

Hence from equations (18) and (19), we have

$$
\begin{aligned}
& \mathrm{R}(\mathrm{X}, \mathrm{Y}) \mathrm{Z}=-\frac{1}{\mathrm{n}-3}[\mathrm{~S}(\mathrm{X}, \mathrm{Z}) \mathrm{Y}-\mathrm{S}(\mathrm{Y}, \mathrm{Z}) \mathrm{X} \\
& +\mathrm{g}(\mathrm{X}, \mathrm{Z}) \mathrm{QY}-\mathrm{g}(\mathrm{Y}, \mathrm{Z}) \mathrm{QX}-\mathrm{S}(\mathrm{X}, \mathrm{Z}) \eta(\mathrm{Y}) \xi \\
& +\mathrm{S}(\mathrm{Y}, \mathrm{Z}) \eta(\mathrm{X}) \xi-\eta(\mathrm{X}) \eta(\mathrm{Z}) \mathrm{QY}+\eta(\mathrm{Y}) \eta(\mathrm{Z}) \mathrm{QX}] \\
& +\frac{\mathrm{K}-2}{\mathrm{n}-3}[\mathrm{~g}(\mathrm{X}, \mathrm{Z}) \mathrm{Y}-\mathrm{g}(\mathrm{Y}, \mathrm{Z}) \mathrm{X}] \\
& -\frac{\mathrm{K}}{\mathrm{n}-3}[\mathrm{~g}(\mathrm{X}, \mathrm{Z}) \eta(\mathrm{Y}) \xi-\mathrm{g}(\mathrm{Y}, \mathrm{Z}) \eta(\mathrm{X}) \xi \\
& +\eta(\mathrm{X}) \eta(\mathrm{Z}) \mathrm{Y}-\eta(\mathrm{Y}) \eta(\mathrm{Z}) \mathrm{X}] .
\end{aligned}
$$

Taking inner product on both sides of (20) by $W$, we obtain

$$
\begin{aligned}
& ' R(X, Y, Z, W)=-\frac{1}{n-3}[S(X, Z) g(Y, W) \\
& -S(Y, Z) g(X, W)+g(X, Z) S(Y, W) \\
& -g(Y, Z) S(X, W)-S(X, Z) \eta(Y) \eta(W) \\
& +S(Y, Z) \eta(X) \eta(W)-\eta(X) \eta(Z) S(Y, W) \\
& +\eta(Y) \eta(Z) S(X, W)] \\
& +\frac{K-2}{n-3}[g(X, Z) g(Y, W)-g(Y, Z) g(X, W)] \\
& -\frac{K}{n-3}[g(X, Z) \eta(Y) \eta(W)-g(Y, Z) \eta(X) \eta(W) \\
& +\eta(X) \eta(Z) g(Y, W)-\eta(Y) \eta(Z) g(X, W)] .
\end{aligned}
$$

where ' $R(X, Y, Z, W)=g(R(X, Y) Z, W)$.

Setting $W=\xi$ in (21), we get

$$
\begin{aligned}
& \eta(R(X, Y) Z)=\frac{1}{n-3}[2 S(Y, Z) \eta(X) \\
& -2 S(X, Z) \eta(Y)-g(X, Z) S(Y, \xi) \\
& +g(Y, Z) S(X, \xi)+\eta(X) \eta(Z) S(Y, \xi) \\
& -\eta(Y) \eta(Z) S(X, \xi)+2(K-1) g(X, Z) \eta(Y) \\
& -2(K-1) g(Y, Z) \eta(X)] .
\end{aligned}
$$

Using (12) and (16) in (22), we have

$$
\begin{aligned}
0= & S(Y, Z) \eta(X)-S(X, Z) \eta(Y) \\
& +(2-K) g(Y, Z) \eta(X)-(K-2) g(X, Z) \eta(Y) .
\end{aligned}
$$

Replacing $Y$ by $\xi$ in (23), we get

$$
S(X, Z)=(K-2) g(X, Z)+(K-n-1) \eta(X) \eta(Z),
$$

this implies that

$$
S(X, Z)=a g(X, Z)+b \eta(X) \eta(Z),
$$


Some Curvature Properties of D-conformal Curvature Tensor on LP-Sasakian Manifolds

where

$$
\left\{\begin{array}{l}
a=K-2, b=K-n-1 \\
K=\frac{r+2(n-1)}{n-2} .
\end{array}\right.
$$

From (25), the manifold is an $\eta$-Einstein manifold. This completes the proof of the theorem.

\section{Definition 1}

An LP-Sasakian manifold $\left(M^{n}, g\right)$ is said to be Dconformal semi-symmetric if its D-conformal curvature tensor $B$ satisfies the relation

$$
R(X, Y) \cdot B=0,
$$

where $R(X, Y)$ is the curvature operator.

\section{Theorem 2}

An LP-Sasakian manifold $\left(M^{n}, g\right), n>4$ satisfying the condition $R(X, Y) \cdot B=0$ is an $\eta$-Einstein manifold.

Proof: Let us consider a D-conformal semi-symmetric LP-Sasakian manifold $\left(M^{n}, g\right)$. Then we have

$$
(R(X, Y) \cdot B)(U, V) W=0,
$$

which yields

$$
\begin{aligned}
0 & =R(X, Y) B(U, V) W-B(R(X, Y) U, V) W \\
& -B(U, R(X, Y) V) W-B(U, V) R(X, Y) W .
\end{aligned}
$$

Taking $X=\xi$ in (28) and using (13), we obtain

$$
\begin{aligned}
0 & =g(B(U, V) W, Y) \xi-\eta(B(U, V) W) Y \\
& -g(U, Y) B(\xi, V) W+\eta(U) B(Y, V) W \\
& -g(Y, V) B(U, \xi) W+\eta(V) B(U, Y) W \\
& -g(Y, W) B(U, V) \xi+\eta(W) B(U, V) Y .
\end{aligned}
$$

Taking inner product on both sides of (29) by $\xi$, we get

$$
\begin{aligned}
0= & -g(B(U, V) W, Y)-\eta(B(U, V) W) \eta(Y) \\
& -g(U, Y) \eta(B(\xi, V) W)+\eta(U) \eta(B(Y, V) W) \\
& -g(Y, V) \eta(B(U, \xi) W)+\eta(V) \eta(B(U, Y) W) \\
& -g(Y, W) \eta(B(U, V) \xi)+\eta(W) \eta(B(U, V) Y) .
\end{aligned}
$$

Putting $Y=U$ in (30), we get

$$
\begin{aligned}
0= & g(B(U, V) W, U)+g(U, U) \eta(B(\xi, V) W) \\
& +g(U, V) \eta(B(U, \xi) W)-\eta(V) \eta(B(U, U) W) \\
& +g(U, W) \eta(B(U, V) \xi)-\eta(W) \eta(B(U, V) U) .
\end{aligned}
$$

Now, from (18), we have

$$
\begin{aligned}
& \eta(B(U, V) W)=\frac{2}{n-3}[S(U, W) \eta(V) \\
& -S(V, W) \eta(U)+(K-2)\{g(V, W) \eta(U) \\
& -g(U, W) \eta(V)\}] \text {, } \\
& \eta(B(U, V) \xi)=0 \text {, } \\
& \eta(B(\xi, V) W)=\frac{2}{n-3}[S(V, W)-(K-2) g(V, W) \\
& +(n-K+1) \eta(V) \eta(W)], \\
& \eta(B(U, \xi) W)=-\frac{2}{n-3}[S(U, W)-(K-2) g(U, W) \\
& -(K-n-1) \eta(U) \eta(W)], \\
& \eta(B(U, U) W)=0, \\
& \eta(B(U, V) U)=\frac{2}{n-3}[S(U, U) \eta(V) \\
& -S(U, V) \eta(U)+(K-2)\{g(U, V) \eta(U) \\
& -g(U, U) \eta(V)\}] \text {. }
\end{aligned}
$$

Using (33) and (36) in (31), we obtain

$$
\begin{aligned}
0= & g(B(U, V) W, U)+g(U, U) \eta(B(\xi, V) W) \\
& +g(U, V) \eta(B(U, \xi) W)-\eta(W) \eta(B(U, V) U) .
\end{aligned}
$$

Let $\left\{e_{i}: i=1,2, \ldots, n\right\}$ be an orthonormal basis of the tangent space at any point of the manifold. Putting $U=e_{i}$ in (38) and taking summation for $1 \leq i \leq n$, we get

$$
\begin{aligned}
0 & =\sum_{i=1}^{n} g\left(B\left(e_{i}, V\right) W, e_{i}\right)+(n-1) \eta(B(\xi, V) W) \\
& -\sum_{i=1}^{n} \eta(W) \eta\left(B\left(e_{i}, V\right) e_{i}\right)
\end{aligned}
$$

since $\sum_{i=1}^{n} g\left(e_{i}, V\right) \eta\left(B\left(e_{i}, \xi\right) W\right)=-\eta(B(\xi, V) W)$. From (18), we also have

$$
\begin{aligned}
& \sum_{i=1}^{n} g\left(B\left(e_{i}, V\right) W, e_{i}\right)=\frac{1}{n-3}[K(n-2) \\
& -2(n-1)-r] g(V, W)+\frac{1}{n-3}[r-2(n-1) \\
& -K(n-2)] \eta(V) \eta(W)
\end{aligned}
$$

and

$\sum_{i=1}^{n} \eta(W) \eta\left(B\left(e_{i}, V\right) e_{i}\right)=\frac{2 r+(n-1)(3-2 K)}{n-3} \eta(V) \eta(W)$.

Using (40), (41) and (34) in (39), we obtain 


$$
\begin{aligned}
& S(V, W)=\left[\frac{r+n K-2 n+2}{2(n-1)}\right] g(V, W) \\
& +\left[\frac{r-2 n^{2}+n+(n-2) K-3}{2(n-1)}\right] \eta(V) \eta(W),
\end{aligned}
$$

this implies

$$
S(V, W)=\alpha g(V, W)+\beta \eta(V) \eta(W),
$$

where $\alpha=\left[\frac{r+n K-2 n+2}{2(n-1)}\right]$ and

$$
\beta=\left[\frac{r-2 n^{2}+n+(n-2) K-3}{2(n-1)}\right] .
$$

The relation (43) implies that the manifold is an $\eta$-Einstein manifold. Hence the theorem is proved.

\section{Definition 2}

An LP-Sasakian manifold of dimension $n$ is said to be $\xi$-D-conformally flat if its D-conformal curvature tensor $B(X, Y) Z$ satisfies the relation $B(X, Y) \xi=0$.

\section{Theorem 3}

A $\xi$-D-conformally flat LP-Sasakian manifold $\left(M^{n}, g\right)$ is an $\eta$-Einstein manifold.

Proof: Using equations (1) and (4) in the definition of D-conformal curvature tensor $B(X, Y) Z$ we have

$$
\begin{aligned}
& B(X, Y) \xi=R(X, Y) \xi+\frac{1}{n-3}[S(X, \xi) Y-S(Y, \xi) X \\
& +2 \eta(X) Q Y-2 \eta(Y) Q X \\
& -S(X, \xi) \eta(Y) \xi+S(Y, \xi) \eta(X) \xi] \\
& +\frac{2(K-1)}{n-1}[\eta(Y) X-\eta(X) Y] .
\end{aligned}
$$

By virtue of (14) and (16), (44) reduces to

$$
\begin{aligned}
& B(X, Y) \xi=\frac{2}{n-3}[(K-2)\{\eta(Y) X-\eta(X) Y\} \\
& +\eta(X) Q Y-\eta(Y) Q X] .
\end{aligned}
$$

From the definition of $\xi$-D-conformally flat and the relation (45), we obtain

$$
(K-2)\{\eta(Y) X-\eta(X) Y\}+\eta(X) Q Y-\eta(Y) Q X=0 .
$$

Taking inner product on both sides of (46) by $W$, we get

$$
\begin{aligned}
0 & =(K-2)\{\eta(Y) g(X, W)-\eta(X) g(Y, W)\} \\
& +\eta(X) S(Y, W)-\eta(Y) S(X, W) .
\end{aligned}
$$

Putting $Y=\xi$ in (47) and using (1), (4) and (16), we obtain

$$
\begin{aligned}
0 & =(K-2)\{-g(X, W)-\eta(X) \eta(W)\} \\
& +(n-1) \eta(X) \eta(W)+S(X, W)
\end{aligned}
$$

which yields

$$
\begin{gathered}
S(X, W)=a g(X, W)+b \eta(X) \eta(W),(49) \text { where } \\
a=K-2, b=K-n-1 \text { and } K=\frac{r+2(n-1)}{n-2} .
\end{gathered}
$$

From (49) it is clear that the manifold is $\eta$-Einstein.

This completes the proof of our theorem.

\section{Definition 3}

An LP-Sasakian manifold $\left(M^{n}, g\right)$ is said to be $\varphi$-Dconformally flat if its D-conformal curvature tensor $B(X, Y) Z$ satisfies the relation

$\varphi^{2}(B(\varphi X, \varphi Y) \varphi Z)=0$ or equivalently

$$
g(B(\varphi X, \varphi Y) \varphi Z, \varphi W)=0
$$

for any vector fields $X, Y, Z, W \in T_{p}(M)$.

\section{Theorem 4}

A $\varphi$-D-conformally flat LP-Sasakian manifold $\left(M^{n}, g\right)$ is an $\eta$-Einstein manifold.

Proof: Let us consider an LP-Sasakian manifold $\left(M^{n}, g\right)$ which is $\varphi$-D-conformally flat, then by the definition of $\varphi$-D-conformally flat we have

$$
g(B(\varphi X, \varphi Y) \varphi Z, \varphi W)=0 .
$$

From (7) and the definition of D-conformal curvature tensor we have

$$
\begin{aligned}
& g(B(\varphi X, \varphi Y) \varphi Z, \varphi W)=g(R(\varphi X, \varphi Y) \varphi Z, \varphi W) \\
& +\frac{1}{n-3}[S(\varphi X, \varphi Z) g(\varphi Y, \varphi W)-S(\varphi Y, \varphi Z) g(\varphi X, \varphi W) \\
& +g(\varphi X, \varphi Z) g(Q \varphi Y, \varphi W)-g(\varphi Y, \varphi Z) g(\varphi \varphi X, \varphi W)] \\
& -\frac{K-2}{n-3}[g(\varphi X, \varphi Z) g(\varphi Y, \varphi W)-g(\varphi Y, \varphi Z) g(\varphi X, \varphi W)] .
\end{aligned}
$$

Using (50) in (51), we get

$$
\begin{aligned}
& 0=g(R(\varphi X, \varphi Y) \varphi Z, \varphi W)+\frac{1}{n-3}[S(\varphi X, \varphi Z) g(\varphi Y, \varphi W) \\
& -S(\varphi Y, \varphi Z) g(\varphi X, \varphi W)+g(\varphi X, \varphi Z) g(Q \varphi Y, \varphi W) \\
& -g(\varphi Y, \varphi Z) g(Q \varphi X, \varphi W)]-\frac{K-2}{n-3}[g(\varphi X, \varphi Z) g(\varphi Y, \varphi W) \ldots(52) \\
& -g(\varphi Y, \varphi Z) g(\varphi X, \varphi W)] .
\end{aligned}
$$


In view of (3), (12) and (17) in (52), we obtain

$$
\begin{aligned}
& g(R(X, Y) Z, W)+g(Y, Z) \eta(X) \eta(W) \\
& +g(X, W) \eta(Y) \eta(Z)-g(X, Z) \eta(Y) \eta(W) \\
& -g(Y, W) \eta(X) \eta(Z)+\frac{1}{n-3}[S(X, Z) g(Y, W) \\
& -S(Y, Z) \eta(X) \eta(W)+(n-1) g(Y, W) \eta(X) \eta(Z) \\
& -(n-1) g(X, W) \eta(Y) \eta(Z)+S(Y, W) g(X, Z) \\
& +S(Y, W) \eta(X) \eta(Z)-S(X, W) \eta(Y) \eta(Z) \\
& -S(Y, Z) g(X, W)+(n-1) g(X, Z) \eta(Y) \eta(W) \\
& -S(X, W) g(Y, Z)+S(X, Z) \eta(Y) \eta(W) \\
& -(n-1) g(Y, Z) \eta(X) \eta(W)] \\
& -\frac{K-2}{n-3}[g(X, Z) g(Y, W)-g(Y, Z) g(X, W) \\
& -g(Y, Z) \eta(X) \eta(W)+g(X, Z) \eta(Y) \eta(W) \\
& +g(Y, W) \eta(X) \eta(Z)-g(X, W) \eta(Y) \eta(Z)]=0
\end{aligned}
$$

where

$$
g(R(X, Y) Z, W)=g(Y, Z) g(X, W)-g(X, Z) g(Y, W) .
$$

Let $\left\{e_{i}: i=1,2, \ldots, n\right\}$ be an orthonormal basis of the tangent space at any point of the manifold. Putting $X=W=e_{i}$ in (53) and taking summation over $i, 1 \leq i \leq n$, we get

$$
\begin{aligned}
& S(Y, Z)=\left\{\frac{n K-2 n-r-2}{2}\right\} g(Y, Z) \\
& +\left\{\frac{n K-2 K-6 n-r+14}{2}\right\} \eta(Y) \eta(Z)
\end{aligned}
$$

which implies

$$
S(Y, Z)=A g(Y, Z)+B \eta(Y) \eta(Z)
$$

where $A=\left\{\frac{n K-2 n-r-2}{2}\right\}$ and

$$
B=\left\{\frac{n K-2 K-6 n-r+14}{2}\right\} \text {. }
$$

From relation (55), we conclude that the manifold is $\eta$ Einstein. This proves the theorem.

\section{ACKNOWLEDGEMENT}

The author is grateful to Prof. Dr. Chet Raj Bhatta for his valuable comments and suggestions towards the improvement of this paper.

\section{REFERENCES}

Bagewadi, C. S., Venkatesha and Basavaraja-ppa, N. S. 2008. On LP-Sasakian mani-folds, Scientia Series A Mathematical Sciences 16: 1-8.

Chuman, G. 1983. On the D-conformal curva-ture tensor, Tensor N. S. 46: 125-129.

De, U. C., Matsumoto, K. and Shaikh, A. A. 1999. On Lorentzian para-Sasakian mani-folds, Rendicontidel SeminarioMatematico di Messina, Serie II, Supplemento al. 3: 149-158.

De, U. C., Shaikh, A. A. and Sengupta, A. 2002. On LPSasakian manifolds with a coefficient $\alpha$, Kyungpook Math. J. 42: 177-186.

Ikawa, T. and Erdogan, M. 1996. Sasakian manifolds with Lorentzian metric, Kyungpook Math. J. 35: 517-526.

Ikawa, T. and Jun, J. B. 1997. On sectional curvature of a normal contact Lorent-zian manifold, Korean Journal Math. Sci. 4: 27-33.

Matsumoto, K. 1989. On Lorentzian para-contact manifolds, Bull. of Yamagata Univ., Nat. Sci. 12: 151-156.

Matsumoto, K. and Mihai, I. 1988. On a certain transformation in a Lorentzian para-Sasakian manifold, Tensor N. S. 47: 189-197.

Mihai, I. and Rosca, R. 1992. On Lorentzian P-Sasakian manifolds, Classical Analysis, World scientific publication, 155-169.

Shaikh, A. A. and Biswas, S. 2004. On LP-Sasakian manifolds, Bull. Malaysian Sc. Soc. 27: 17-26.

Szabo, Z. I. 1982. Structure theorems on Riemannian spaces satisfying $R(X, Y) \cdot R=0$, I, The local version, J. Diff. Geom. 17: 531-582.

Tripathi, M. M. and De, U. C. 2001. Lore-ntzian almost paracontact manifolds and their submanifolds, $J$. of the Korean Society Math. Education 2: 101-125. 